\title{
Relaxational dynamics in the glassy, supercooled liquid, and orientationally disordered crystal phases of a polymorphic molecular material
}

\author{
M. Jiménez-Ruiz, M. A. González, and F. J. Bermejo \\ Instituto de Estructura de la Materia, Consejo Superior de Investigaciones Científicas, Serrano 123, E-28006 Madrid, Spain \\ M. A. Miller and Norman O. Birge \\ Department of Physics and Astronomy and Center for Fundamental Materials Research, Michigan State University, \\ East Lansing, Michigan 48824 \\ I. Cendoya and A. Alegría \\ Departamento de Física de Materiales, Facultad de Química, Apartado 1072, 20080 San Sebastián, Spain
}

(Received 14 September 1998)

\begin{abstract}
The relaxational dynamics of the ambient pressure phases of ethyl alcohol are studied by means of measurements of frequency dependent dielectric susceptibility. A comparison of the $\alpha$ relaxation in the supercooled liquid and in the rotator phase crystal indicates that the molecular rotational degrees of freedom are the dominant contribution to structural relaxation at temperatures near the glass transition, the flow processes having lesser importance. Below the glass transition a secondary $\beta$ relaxation is resolved for the orientational and structural glasses. Computer molecular-dynamics results suggest that localized molecular librations, strongly coupled to the low-frequency internal molecular motions, are responsible for this secondary relaxation. [S0163-1829(99)00914-5]
\end{abstract}

\section{INTRODUCTION}

The dynamics of glass-forming materials in the highly viscous regime characteristic of the supercooled liquid have been extensively studied during recent years from theoretical and experimental points of view. Empirically, some regularities have been found in a vast number of glasses, which lead to their identification as fingerprints of glassy dynamics.

Mechanical or dielectric spectroscopies are sensitive to motions with frequencies stretching from macroscopic to mesoscopic scales. This makes them especially suited for the exploration of the dynamics of glass-forming liquids within their highly viscous regimes (i.e., shear viscosities about $\left.10^{2}-10^{10} \mathrm{P}\right)$. The strongest peak in the dielectric spectrum known as the $\alpha$ relaxation shows some features regarded as "universal." These include a non-Debye behavior of its line shape and a temperature dependence of the relaxation time $\tau(T)$ strongly departing from Arrhenius as the temperature is decreased towards the glass transition temperature $T_{\mathrm{g}}$, and showing a divergence at temperatures below $T_{\mathrm{g}}$. Such a dependence is usually parameterized by means of the empirical Vogel-Tamman-Fulcher law. ${ }^{1}$ Secondary, $\beta$, or sub- $T_{\mathrm{g}}$ relaxations are detectable at higher frequencies, usually but not exclusively at temperatures well below $T_{\mathrm{g}}$. They commonly show Arrhenius behavior and have been measured for many glassy systems, albeit reliable data are scarce since the signals are very weak and broad. Whether this relaxation arises from intramolecular motions which remain active below $T_{\mathrm{g}}$, a view defended by $\mathrm{Wu}^{2}$ and others, or it is an intrinsic property of glasses due to local rearrangements taking place within some minima of the potential energy, ${ }^{3}$ still needs to be clarified.
Most studies to date have been conducted on materials that can be studied within the supercooled liquid (SCL) state for a reasonable long period of time. This excludes simple binary alloys, which may be described in terms of soft spheres. Instead, materials with a complexity ranging from ionics to high polymers have been scrutinized ${ }^{4}$ and the results have sometimes been interpreted within the framework of theories couched for simple hard-sphere fluids. This problem is only recently being addressed with the introduction of mode coupling approximations which take explicit account of the nonsphericity of the particles ${ }^{5}$ and orientation dependent forces.

While the rotational dynamics in low viscosity molecular liquids is usually understood on hydrodynamic grounds (Stokes-Einstein-Debye) ${ }^{6}$ such approximations usually break down within the SCL regime. This happens as a consequence of the sluggish motions which take place when the shear viscosity exceeds some tens of poises, which makes any description in terms of uncoupled rotations and centerof-mass diffusion of scarce value. In actual fact, on strict hydrodynamic grounds, the same dependence on viscosity (and temperature) is expected for both mass diffusion and rotational coefficients. Therefore a test of the hydrodynamic predictions could be carried out by studying a single system where one of the two kinds of motions can be cleanly isolated by experimental means.

As described previously, ethyl alcohol can be prepared in two phases showing quantitatively close "glassy" behaviors at the same temperatures and very close densities, one of these phases with topological disorder (liquid and glassy) and the other showing orientational disorder (rotator crystal and orientational glass phases). ${ }^{7}$ By orientationally disordered crystals, we refer to those crystalline solids where the 
molecular centers of mass sit at lattice positions of a longrange periodic array, while molecular orientations remain disordered. Such disorder may be of dynamic nature as happens in the rotator phase crystal (RP) where the molecules execute whole body rotations, ${ }^{8,9}$ or static as in the orientational glass $(\mathrm{OG})$ state which is attained by freezing the rotations into random orientations. Both phases thus provide a way to separate the contributions of the rotational and centerof-mass motions to the dynamics.

The $\mathrm{RP} \rightarrow \mathrm{OG}$ rotational freezing transition exhibits most of the characteristics of a purely dynamical phenomenon. It shows a jump in the specific heat across the transition ${ }^{10}$ concomitant with a jump in the thermal expansivity, ${ }^{11}$ without any other change attributable to a structural transition. Also and in parallel with the canonical liquid $\rightarrow$ glass, the transition can be induced by application of moderate pressures. ${ }^{12}$

To investigate in more detail the close similarity between the relaxation processes of ethanol in the amorphous and orientational glass form, low-temperature specific-heat measurements and neutron time-of-flight (TOF) spectroscopy have been carried out, and the results reveal that at low temperature the vibrational density of states of these two phases is very similar. ${ }^{14}$ Such dynamic proximity has also been extended to macroscopic scales by means of dielectric spectroscopy measurements, ${ }^{15}$ and the results lead to the consideration of this material as an ideal candidate for studies of the glass transition.

Here, our purpose is to provide as detailed a comparison as possible of the relaxational processes taking place at both sides of the glass transitions in glassy and orientationally disordered crystalline ethanol by means of dielectric spectroscopy. Our aim goes well beyond that of our previous report $^{15}$ since a far wider spectral band, comprising both $\alpha$ and $\beta$ relaxations is considered. Since preliminary data ${ }^{15}$ indicated a close analogy between the main relaxation appearing in both SCL and RP phases, here we pursue a study of the relaxation detectable below freezing in both solids as well as of the high-frequency wing of the spectra of both SCL and RP. The motivation behind this is the possibility offered by such a chemically simple material to unravel some of the details of the dynamics at microscopic scales which lead to the observation of macroscopic relaxations, by means of the concurrent use of experimental and computer simulation approaches.

\section{EXPERIMENTAL AND COMPUTATIONAL DETAILS}

Measurements of the frequency and temperaturedependent complex dielectric susceptibility were carried out using three different schemes depending upon the frequency range under consideration. In the range $1 \mathrm{mHz}-10 \mathrm{kHz}$, the in-phase and out-of-phase currents through the capacitor were measured using a Stanford SR850 dual phase lock-in amplifier together with a Stanford SR570 current-to-voltage amplifier. From frequencies of $1 \mathrm{kHz}-10 \mathrm{MHz}$, a HewlettPackard 4192A impedance analyzer was used in a standard four-terminal pair configuration to measure the capacitance and conductance of the sample. From $1 \mathrm{MHz}$ to $1 \mathrm{GHz}$ a Hewlett-Packard 4191A rf impedance analyzer was used, with a coaxial measurement line.

The sample material consisted of 200-proof, dehydrated ethyl alcohol (ethanol) obtained from Quantum Chemical Company of Tuscola, Illinois. To avoid contamination with water vapor, filling of the capacitor was carried out at room temperature in a dry nitrogen atmosphere. For the lowtemperature measurements $(T<110 \mathrm{~K})$ a sealed coaxial capacitor identical in design to that used in our previous work ${ }^{16}$ was used. After filling the capacitor, the sample cell was frozen, evacuated of remaining nitrogen gas, and sealed. The sample cell was then mounted inside a nested cryocan arrangement described elsewhere. ${ }^{16}$ Finally, the entire cryocan assembly was immersed in a double-walled glass dewar. This design provided a temperature control within $0.05 \mathrm{~K}$ over the range 4-300 K, and minimized the occurrence of temperature gradients across the sample. For the measurements at $T>150 \mathrm{~K}$, in the liquid state, and high frequencies, $1 \mathrm{MHz}-1 \mathrm{GHz}$, a parallel plate sample capacitor was mounted as part of the inner conductor of the coaxial measurement line. The temperature control in this case was achieved by employing a nitrogen-jet heating/cooling system with temperature stability better than $0.1 \mathrm{~K}$.

The sample preparation regarding the normal glass phase and the rotator phase (RP) crystal is straightforward since it only involves a quench below $T_{\mathrm{g}}=97 \mathrm{~K}$ at a rate greater than $6 \mathrm{~K} / \mathrm{min}$ (glass) and a subsequent annealing at $110 \mathrm{~K}$ during half an hour (RP). The orientational glass (OG) is formed from the RP by cooling down below $T_{\mathrm{g}}^{\mathrm{OG}}=97 \mathrm{~K}$. The measurements of the $\alpha$ relaxation span the frequency range from $1 \mathrm{mHz}-10 \mathrm{MHz}$, of the liquid state from 1 $\mathrm{MHz}-1 \mathrm{GHz}$, and the $\beta$ relaxation from $10 \mathrm{~Hz}-10 \mathrm{MHz}$.

A number of computer simulations of the glass, supercooled liquid, and crystalline modifications of the material have been carried out. ${ }^{17}$ The results relevant for this work are those obtained from a simulation using the optimized potential model for liquid simulations (OPLS) ${ }^{18}$ which describes reasonably well the static and some dynamic properties of the normal-liquid phase. ${ }^{19}$ The system consisted of 216 molecules at densities of $0.941 \mathrm{~g} \mathrm{~cm}^{-3}$ and a temperature of 80 $\mathrm{K}$ which corresponds to the glass phase, and $0.888 \mathrm{~g} \mathrm{~cm}^{-3}$ and $180 \mathrm{~K}$, which, within the computer model corresponds to the SCL. The initial configuration was obtained from a quench of the liquid at $298 \mathrm{~K}$ at a cooling rate of $d T / d t$ $=0.1 \mathrm{~K} \mathrm{ps}^{-1}$. Additional details about the model and the simulations (of the liquid, supercooled liquid, and glass) will be given elsewhere. ${ }^{17}$

The functions evaluated from the computed trajectory which are relevant for the present study are the standard orientational correlation functions for a vector fixed to some molecular frame. Two choices were made. One of them locates the vector parallel to the molecular dipole moment $\left(\boldsymbol{\mu}=2.22 \mathrm{D} \approx 7.405 \times 10^{-30} \mathrm{C} \mathrm{m}\right)$, whereas the other positions it along the $\mathrm{O}-\mathrm{H}$ molecular bond. Also, the Fourier transforms of the angular velocity autocorrelation of the $\mathrm{OH}$ and $\boldsymbol{\mu}$ vectors $\left[P_{\mu}(\omega)\right.$ and $\left.P_{\mathrm{OH}}(\omega)\right]$ were evaluated. For a solid or a highly viscous liquid these functions provide a measure of the rotational components of the weighted vibrational density of states, and therefore can be related to measurements of the experimental $Z(\omega)$ frequency distribution derived from neutron inelastic scattering. ${ }^{10,12}$

\section{RESULTS}

In what follows the results concerning the two spectral regions are described separately. 


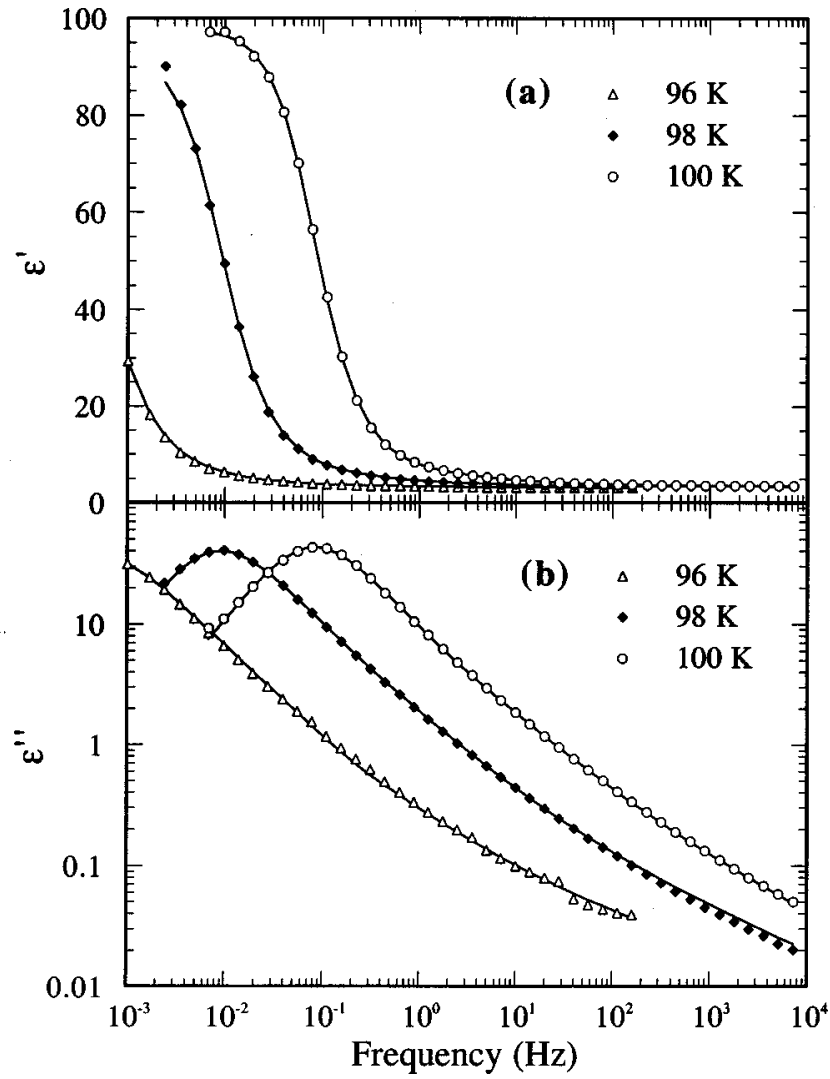

FIG. 1. The real (a) and imaginary (b) parts of the dielectric susceptibility vs frequency at temperatures of $96 \mathrm{~K}$ (glass), $98 \mathrm{~K}$, and $100 \mathrm{~K}$ (supercooled liquid). The solid lines are fits to two Cole-Davidson functions with different relaxation times.

\section{A. $\alpha$ relaxation}

In our previous report ${ }^{15}$ the main relaxational processes in both phases were compared in terms of the real and imaginary parts of the dielectric susceptibility, which were fitted simultaneously to the empirical Cole-Davidson form. ${ }^{20} \mathrm{Al}-$ though this provides good fits for the main relaxation, the model function shows a clear deviation from the experimental data at higher frequencies. In particular, data for the imaginary part of the dielectric susceptibility lie systematically above the Cole-Davidson curves for all frequencies starting about two decades above the peak frequency. Figures 1(a) and 1(b) show the real $\left(\epsilon^{\prime}\right)$ and imaginary $\left(\epsilon^{\prime \prime}\right)$ parts of the susceptibility of the supercooled liquid (SCL) for temperatures of 96, 98, and $100 \mathrm{~K}$. Figures 2(a) and 2(b) show the corresponding quantities for the rotator phase (RP) crystal for temperatures from 96 to $108 \mathrm{~K}$. The logarithmic plots of $\epsilon^{\prime \prime}$ show a broad tail starting about two decades above the peak frequency and extending to the highest explored frequencies. We have followed two different routes to interpret this tail. The first considers that the susceptibility curves shown in Figs. 1 and 2 are characterized by a single relaxation whose shape is nearly universal, as first suggested by Dixon et al. ${ }^{21}$ Those authors showed that dielectric data from several different materials at many temperatures could be made to coincide when plotted on a particular type of logarithmic scaling plot. The "universal" curve they found on the scaling plot corresponds to the features seen in raw data such as that shown in Figs. 1 and 2, or from other

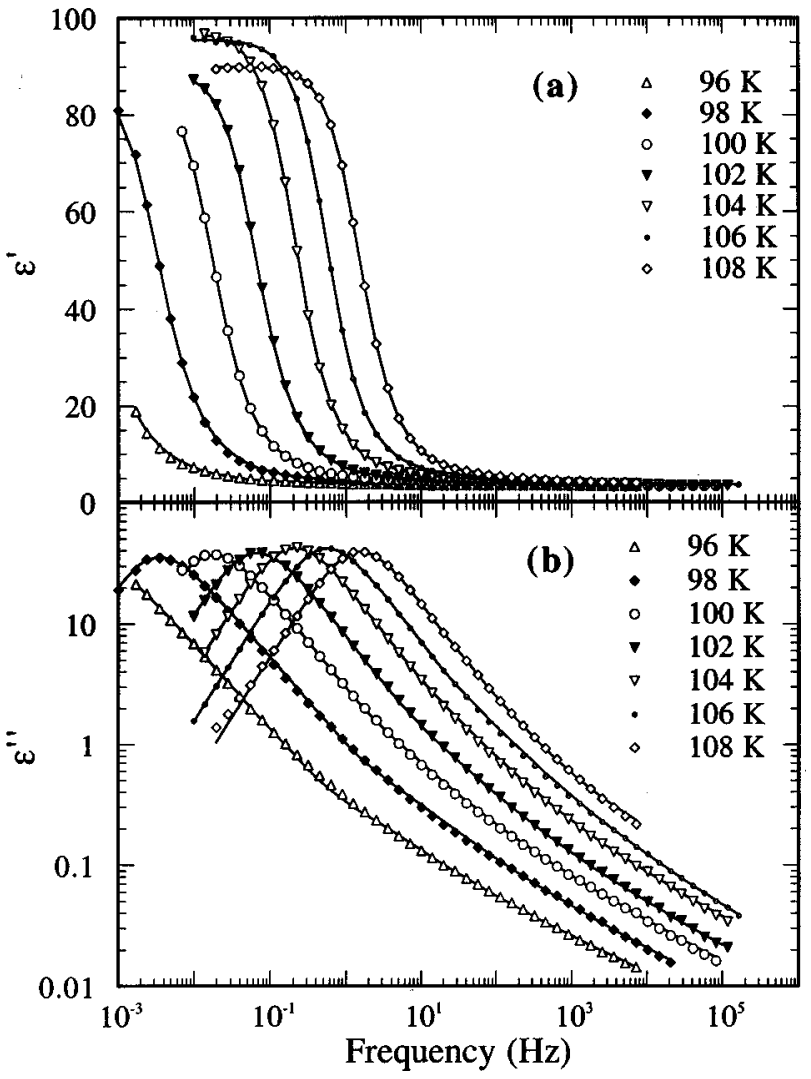

FIG. 2. The real (a) and imaginary (b) parts of the dielectric susceptibility as a function of frequency at temperatures of $96 \mathrm{~K}$ (orientational glass) and from 98-108 K (rotator phase crystal). The solid lines are fits to two Cole-Davidson functions.

materials. $^{22}$ In particular, $\epsilon^{\prime \prime}$ grows linearly with frequency below the peak, then decreases with a power law somewhat less than 1 above the peak, and finally flattens out to a more shallow power law starting at a frequency about two decades above the peak. The presence of a universal curve implies certain relationships between the two power laws above the peak, as well as between the peak frequency and the frequency at which the slope changes. Rather than scaling our data onto the "Dixon-Nagel scaling plot," we prefer to examine the raw data on a simple log-log plot where the power laws are most clearly visible. In the search for a reliable way to parameterize the data, we found that the sum of two ColeDavidson functions provides excellent fits to the data over the entire frequency range, while ensuring that the simultaneous fits to $\epsilon^{\prime}$ and $\epsilon^{\prime \prime}$ obey the Kramers-Krönig relationship. The fitting function is given by

$$
\boldsymbol{\epsilon}=\boldsymbol{\epsilon}_{\infty}+\sum_{i=1}^{2} \frac{\left(\epsilon_{0}-\epsilon_{\infty}\right)_{i}}{\left(1-i \omega / \omega_{\mathrm{p} i}^{\mathrm{CD}}\right)^{\alpha_{i}}},
$$

where $\left(\epsilon_{0}-\epsilon_{\infty}\right)_{i}$ is the relaxation strength, $\omega_{\mathrm{p} i}$ is the peak frequency (related to the mean relaxation time, $\tau_{i} \propto \omega_{\mathrm{p} i}^{-1}$ ), $\epsilon_{\infty i}$ is the susceptibility at high frequencies and $\alpha_{i}$ is the width parameter $^{23}$ which usually varies between 0 and 1 . The Debye (Lorentzian) function is approached as $\alpha_{i} \rightarrow 1$.

Using the Kramers-Krönig relationship, the real and imaginary parts of the dielectric susceptibility, $\epsilon^{\prime}(\omega)$ and $\epsilon^{\prime \prime}(\omega)$ are fitted simultaneously: 


$$
\begin{gathered}
\boldsymbol{\epsilon}^{\prime}=\boldsymbol{\epsilon}_{\infty}+\sum_{i=1}^{2}\left(\epsilon_{0}-\epsilon_{\infty}\right)_{i} \frac{\cos \left[\alpha_{i} \arctan \omega \tau_{i}\right]}{\left[1+\omega^{2} \tau_{i}^{2}\right]^{\alpha_{i} / 2}}, \\
\epsilon^{\prime \prime}=\sum_{i=1}^{2} \frac{\left(\epsilon_{0}-\epsilon_{\infty}\right)_{i} \sin \left[\alpha_{i} \arctan \omega \tau_{i}\right]}{\left[1+\omega^{2} \tau_{i}^{2}\right]^{\alpha_{i} / 2}} .
\end{gathered}
$$

The fit determines the seven parameters, $\omega_{\mathrm{p}}, \epsilon_{0}-\epsilon_{\infty}$, and $\alpha$ of the two different relaxations, and $\epsilon_{\infty}$. These parameters fully characterize the shape of the relaxation in the frequency range from $\mathrm{mHz}$ to $\mathrm{MHz}$ and are temperature dependent.

The first Cole-Davidson function fits the main part of the peak, and departs only mildly from a Debye relaxation ( $\alpha$ $\approx 0.8$ ). The second Cole-Davidson function fits the very shallow power law at high frequency, hence it departs strongly from Debye relaxation $(\alpha \approx 0.3)$. To minimize the number of adjustable parameters, the relaxation times $\tau_{i}^{\mathrm{CD}}$ of the Cole-Davidson functions were constrained to be equal. A further examination of the exponents $\alpha_{1}$ and $\alpha_{2}$ shows that they obey the relationship implied by the Dixon-Nagel scaling form. Further details of this analysis can be found in Ref. 24.

An alternative view follows the approach of Garg et al. and others, ${ }^{25-29}$ which analyzed the spectrum of monohydric alcohols in terms of three different dispersion processes. The rationale behind such a treatment is to enable a connection with the high-temperature liquid, where as detailed below, the whole relaxation spectrum can be understood on the basis of phenomenological treatments ${ }^{27-29}$ or fully atomistic simulations. ${ }^{17,19}$ To proceed, we assume that there exist (at least) two different relaxation processes, a main relaxation $(\alpha)$ and a secondary contribution which appears at higher frequencies. Operationally, the only difference between this fitting scheme and the previous one is that now we let $\tau_{2}$ be a free parameter independent of $\tau_{1}$. The real and imaginary parts have thus been fitted to two Cole-Davidson forms given by Eqs. (2) and (3) labeling $i=1$ the main relaxation and $i$ $=2$ the secondary relaxation which could not be tracked at all temperatures within the normal-liquid phase.

Figures 3(a) and 3(b) show the real $\left(\epsilon^{\prime}\right)$ and imaginary $\left(\epsilon^{\prime \prime}\right)$ parts of the dielectric susceptibility of the normal-liquid phase of ethanol at several temperatures between 160 and $270 \mathrm{~K}$. The solid lines represent the fits to two ColeDavidson functions at $160 \mathrm{~K}$ and to one Cole-Davidson from $170-270 \mathrm{~K}$ (only one dispersion process is observed in the frequency range), plus a conductivity term in the imaginary part arising from the sample itself and sample cell which is subtracted [Eq. (3)], $\sigma_{\mathrm{dc}} / \omega$.

The results are summarized below:

(i) Figures 4(a) and 4(b) show the temperature dependence of the Cole-Davidson width parameters of the main and secondary relaxations for both the supercooled liquid (SCL) and rotator phase (RP). The decrease of both $\alpha_{1}$ and $\alpha_{2}$ with decreasing temperature signals a deviation from the Debye behavior. The fact that at the same temperature $\alpha(\mathrm{SCL})>\alpha(\mathrm{RP})$ for both relaxations merits some remarks. The stronger deviation from exponentiallity in the spectra of the RP can be thought of as due to orientational correlations between neighbor units (presumed more important in the RP crystal than in the structural glass ${ }^{8}$ ). The inset of Fig. 4(a) shows the temperature dependence of $\alpha_{1}$ of the liquid phase.

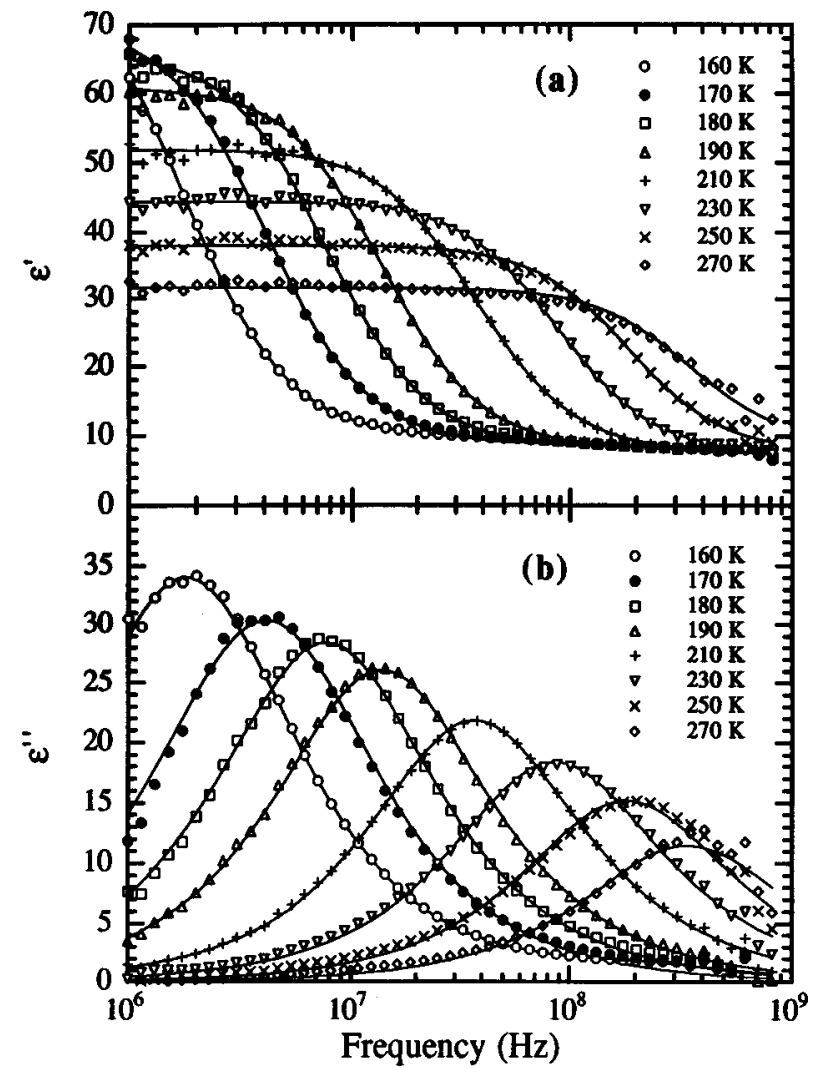

FIG. 3. The real (a) and imaginary (b) parts vs frequency in the liquid phase of ethanol. Solid lines are fits to the Debye function.

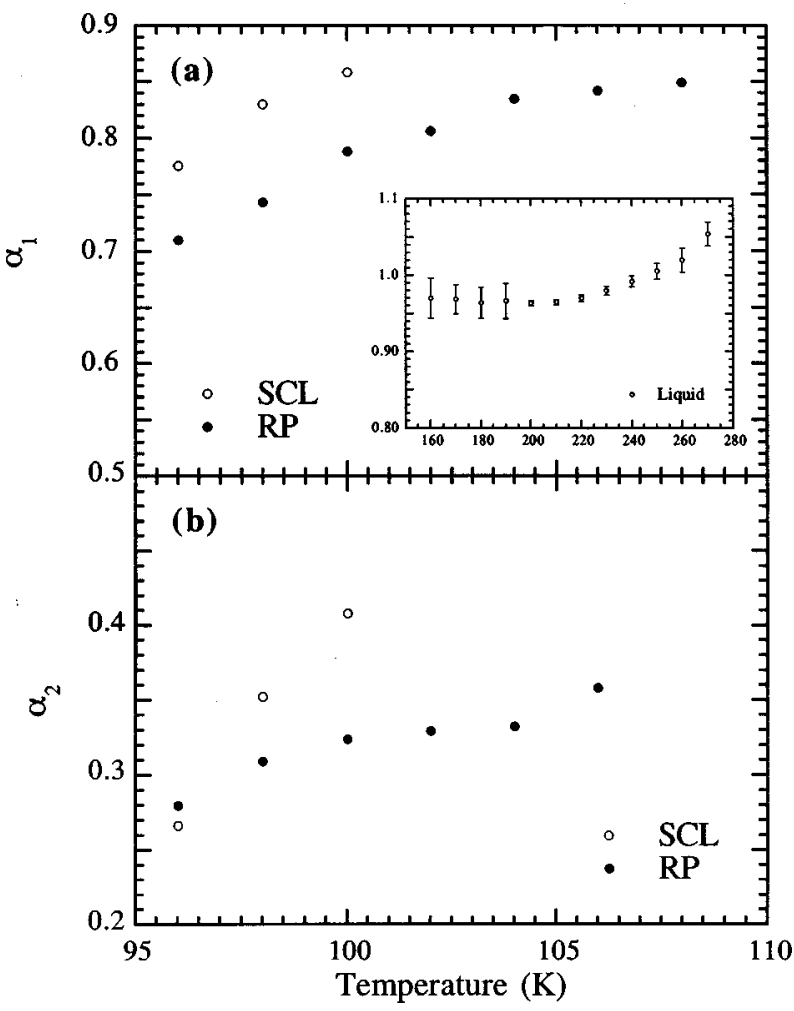

FIG. 4. The temperature dependence of the width parameters $\alpha_{1}$ and $\alpha_{2}$ of the main relaxation for the supercooled liquid $\rightarrow$ glass and rotator phase $\rightarrow$ orientational glass transitions. The inset shows $\alpha_{1}$ for the liquid phase. 


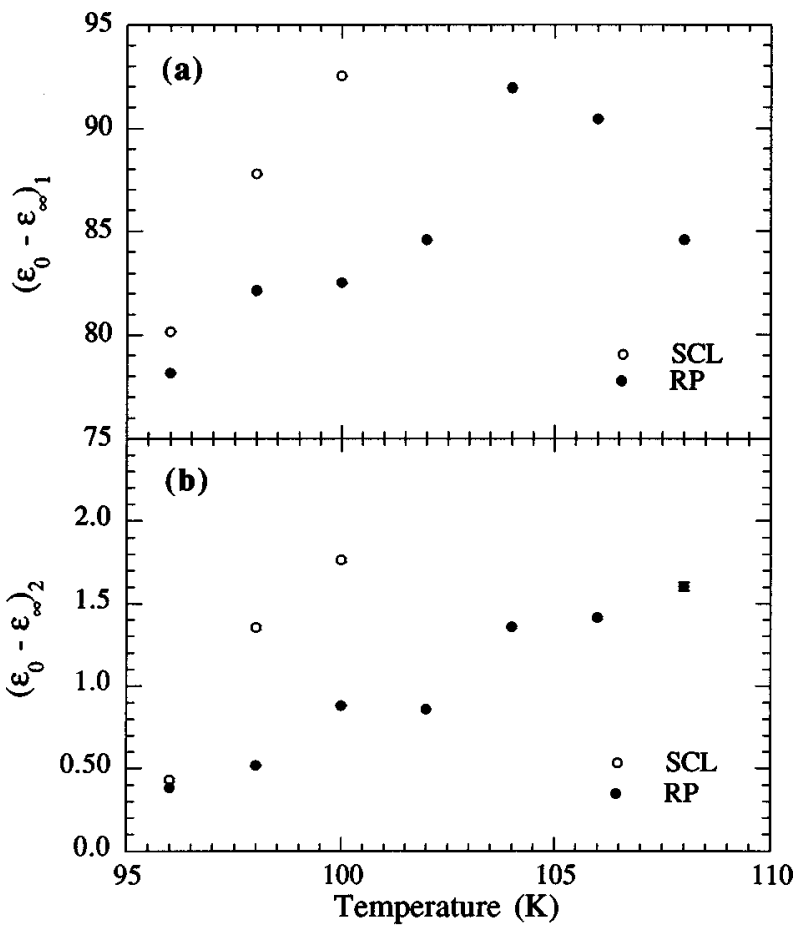

FIG. 5. The temperature dependence of the relaxation strengths $\epsilon_{0}-\epsilon_{\infty}$ of the parts of the main relaxation for the supercooled liquid (SCL) and rotator phase (RP) crystal.

The values are almost equal to 1 , a behavior indistinguishable from isotropic rotational diffusion (Debye).

(ii) Figures 5(a) and 5(b) show the temperature dependence of the the relaxation strength $\epsilon_{0}-\epsilon_{\infty}$ for the two relaxations. The decrease in the relaxation strength below 104 $\mathrm{K}$ in both the RP and SCL data was noted in our previous work. ${ }^{15}$ The decrease cannot be attributed to partial crystallization of the sample because the SCL data were taken on warming of the sample, and the RP data were confirmed to be reproducible on cooling and warming. The data may indicate a slight tendency toward antiparallel alignment of neighboring molecules as the temperature is lowered.

(iii) The dependence of the mean relaxation times $\tau_{1}$ with inverse temperature, obtained from fits to the Cole-Davidson function of data for the RP and SCL phases and to the Debye function in the liquid phase, is shown in Fig. 6. The solid line shows the fit to the Vogel-Tamman-Fulcher form:

$$
\tau=\tau_{0} e^{E_{0} /\left(T-T_{0}\right)},
$$

where $\tau_{0}$ is the attempt relaxation time and $T_{0}$ is the Vogel temperature. The fitting parameters for both SCL and RP phases are summarized in Table I.

Also plotted in Fig. 6 are data for the dependence with temperature of the second relaxation time $\tau_{2}$ corresponding to the high-frequency wing of the $\alpha$ peak if considered as an additional relaxation process (see above). A significant set of data was obtained only for the RP crystal and this is shown in Fig. 6. The point that merits some attention concerns the disparate behavior with temperature of $\tau_{2}$ and the main $\alpha$ relaxation time $\tau_{1}$. The former seems to follow a well defined Arrhenius behavior with an activation energy of 3220 $\mathrm{K}$, in contrast to the Vogel-Fulcher behavior of the latter.

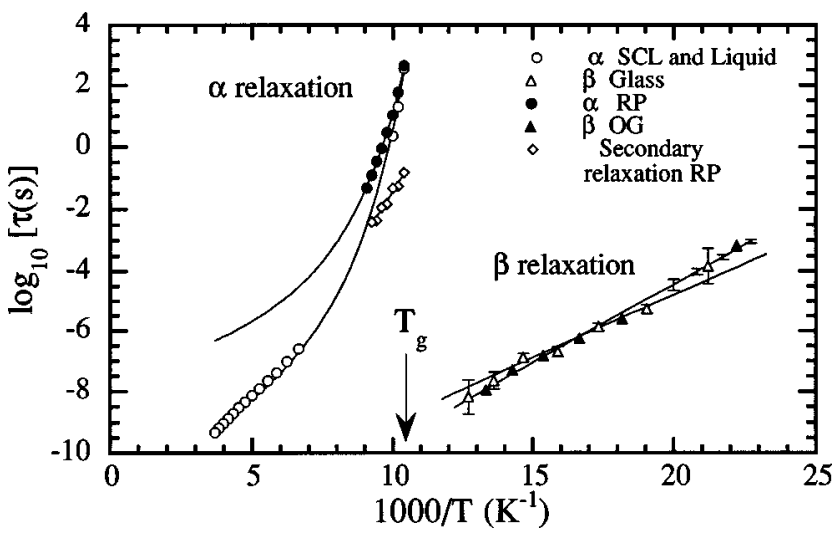

FIG. 6. The mean relaxation times vs $1000 / T$ for the $\alpha$ (liquid phase, SCL, and RP crystal phases), secondary (RP) and $\beta$ (structural glass and OG) relaxations. The solid lines are fits to the VogelTamman-Fulcher form for the $\alpha$ relaxation and to the Arrhenius form for the $\beta$ and secondary ones. The parameters obtained after the fitting are listed in Table I.

Figure 6 also shows the time scale of the $\beta$ relaxation of the corresponding low-temperature phases (structural glass and orientational glass), but further comments are deferred to the next section.

From Fig. 6, it is clearly seen that the relaxation times in the SCL and RP phases are about the same at $T=96 \mathrm{~K}$, and as the temperature increases a shift to a longer relaxation times in the RP relative to the SCL is observed. This behavior can be explained considering the degrees of freedom available through the glass transition in each phase. The $\mathrm{OG} \rightarrow \mathrm{RP}$ transition involves the melting of rotational degrees of freedom, while the glass $\rightarrow$ SCL transition involves rotational and translational degrees of freedom. As the temperature increases above $96 \mathrm{~K}$, the additional translational degrees of freedom present in the SCL provide the molecules with a less restrictive local environment, leading to increasingly shorter relaxation times in the SCL relative to the RP for a given temperature. This interpretation is in agreement with the observed values for the jump in specific heat at both transitions, ${ }^{8}$ the $\mathrm{RP} \rightarrow \mathrm{OG}$ transition involves a $\triangle C_{P}$ of about $22 \mathrm{~J} \mathrm{~mol}^{-1} \mathrm{~K}^{-1}$ and the canonical glass transition SCL $\rightarrow$ glass is accompanied by a jump in $\triangle C_{P}$ of $31 \mathrm{~J} \mathrm{~mol}^{-1} \mathrm{~K}^{-1}$. The extra $9 \mathrm{~J} \mathrm{~mol}^{-1} \mathrm{~K}^{-1}$ can be assigned to the translational degrees of freedom that are not activated in the $\mathrm{OG} \rightarrow \mathrm{RP}$ transition. These results suggest that the rotational degrees of freedom are the dominant contributor to structural relaxation processes in ethanol, whereas mass diffusion associated with translational degrees of freedom in the

TABLE I. Values obtained by fitting the temperature dependence of the mean relaxation time in the RP crystal and SCL phases to the Vogel-Tamman-Fulcher equation, and in the glass and OG to the Arrhenius law. The $f_{0}$ value is defined as $1 /\left(2 \pi \tau_{0}\right)$.

\begin{tabular}{lccccc}
\hline \hline Relaxation & Phase & $\tau_{0}(\mathrm{sec})$ & $f_{0}(\mathrm{~Hz})$ & $E_{0}(\mathrm{~K})$ & $T_{0}$ \\
\hline$\alpha$ & SCL & $2.3 \times 10^{-11}$ & $2.7 \times 10^{11}$ & 693 & 73.1 \\
& RP & $3.2 \times 10^{-8}$ & $2.0 \times 10^{8}$ & 525 & 73.4 \\
$\beta$ & Glass & $6.5 \times 10^{-14}$ & $2.4 \times 10^{12}$ & 941 & 0 \\
& OG & $1.6 \times 10^{-14}$ & $1.0 \times 10^{13}$ & 1203 & 0 \\
\hline \hline
\end{tabular}




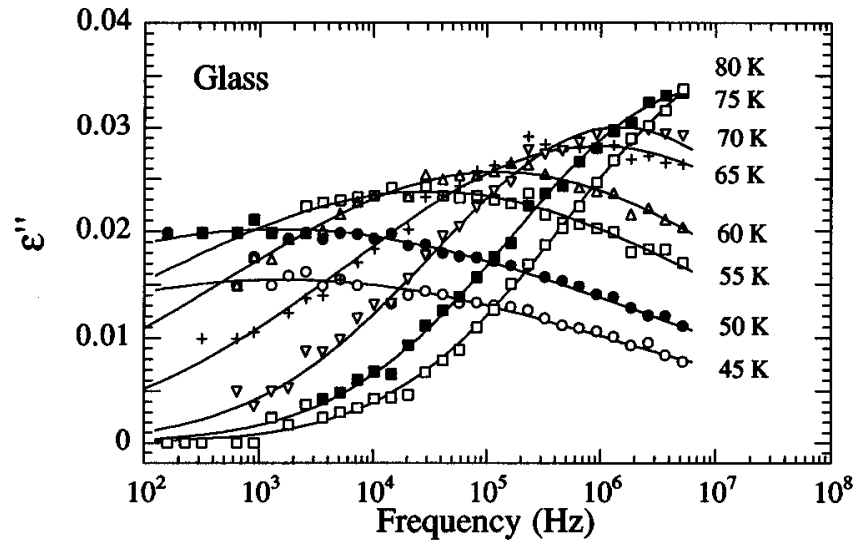

FIG. 7. Imaginary $\epsilon^{\prime \prime}$ part of the dielectric susceptibility of the structural glass phase of ethanol at several temperatures for the $\beta$ relaxation. The continuous lines are fits using Eq. (5).

SCL contributes to a lesser extent. This result is also supported from the values of $T_{0}$ in Table I, almost equal for both phases, which provide an additional indication of the fact that at low temperatures the rotations dominate the dynamics of the SCL.

(iv) As also shown in Fig. 6, the dynamics of the SCL and RP take place at scales which approach each other as motions get progressively frozen. Below $T_{\mathrm{g}}$, motions giving rise to dielectric response should be originated by sequential molecular reorientations. These should be able to survive after whole molecule large-angle rotations freeze out at temperatures below $T_{\mathrm{g}}$ and will give rise to additional relaxational response as described in the next section. This agrees with work of Ramos et al. where they conclude, by means of low-temperature specific-heat measurements and neutron TOF spectroscopy, ${ }^{14}$ that the low-frequency dynamics of the glass and the OG are remarkably close.

\section{B. $\beta$ relaxation}

Figures 7 and 8 show the imaginary parts $\left(\epsilon^{\prime \prime}\right)$ of the susceptibilities for the structural and orientational glass (OG) phases at several temperatures well below the glass transition. Data concerning the real part were too weak to be significant.

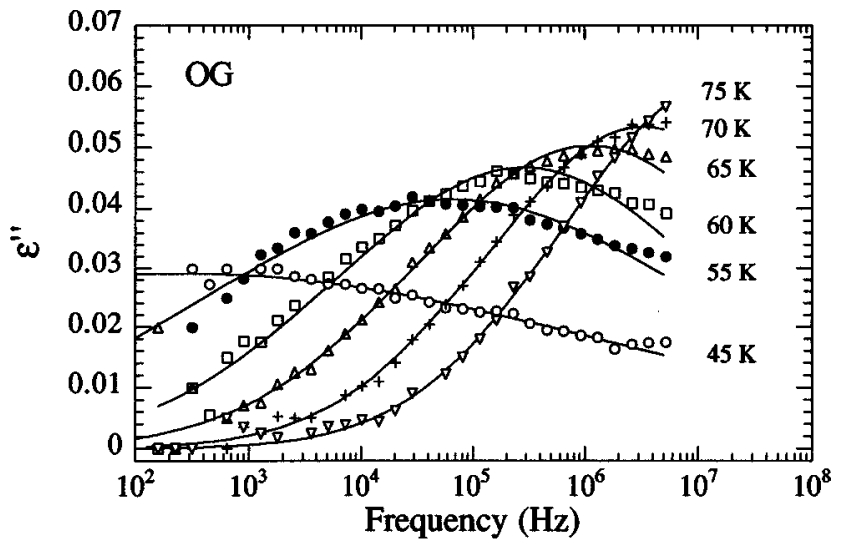

FIG. 8. Imaginary $\epsilon^{\prime \prime}$ part of the dielectric susceptibility of the OG of ethanol for the $\beta$ relaxation. The lines correspond to the fitting to the Eq. (5).

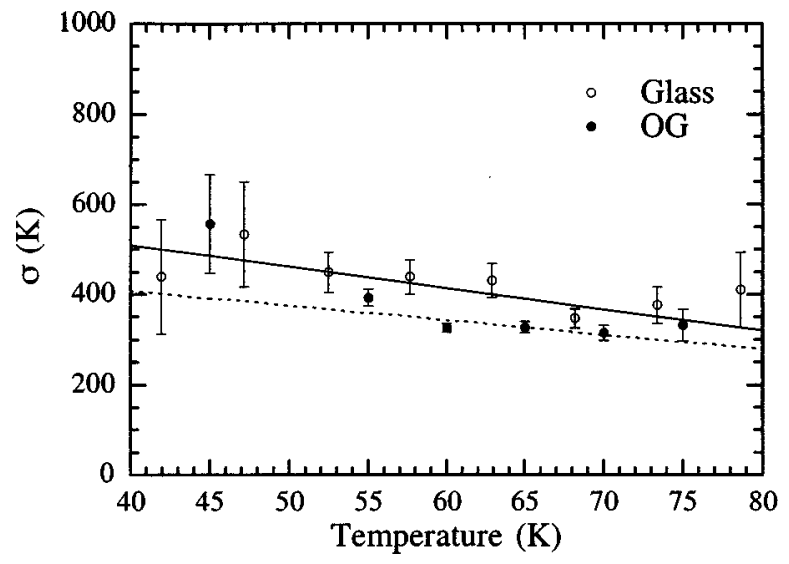

FIG. 9. Comparison of the temperature dependence of the width of the energy barrier distribution between the glass and OG phases of ethanol in the $\beta$ relaxation. The solid and dashed lines are the linear fits of the glass and OG, respectively.

The shape of the $\beta$ relaxation peaks can be described with a log-normal distribution, a form which has been used for some other glassy crystals, ${ }^{30}$ which reads

$$
\epsilon(\omega)=\frac{\epsilon_{0}-\epsilon_{\infty}}{\sqrt{\pi} W} e^{-\frac{\left[\log \omega-\log \omega_{\mathrm{p}}\right]^{2}}{W^{2}}},
$$

where the fitting parameters are the peak frequency of the relaxation, $\omega_{\mathrm{p}}$ (related to the mean relaxation time $\tau$ $\left.=\omega_{\mathrm{p}}^{-1}\right)$; the relaxation strength $\epsilon_{0}-\epsilon_{\infty}$, and the full width at half maximum in decades $W$. Its functional form can be derived if one postulates the presence of a Gaussian distribution of energy barrier heights, ${ }^{30,31}$ and is written as

$$
\begin{aligned}
\epsilon(\omega, T)= & \epsilon_{\infty}+\left(\epsilon_{0}-\epsilon_{\infty}\right) \int d E \frac{1}{\sqrt{\pi} \sigma} \\
& \times e^{-\left(E-E_{0}\right)^{2} / \sigma^{2}} \frac{1}{1-i \omega / \omega_{0} e^{E / K T}} .
\end{aligned}
$$

The width of the energy barrier distribution is given by $\sigma=W K T \ln 10$ and the peak energy $E_{0}$ from the slope of the Arrhenius plot.

(i) Figure 6 shows the temperature dependence of $\log (\tau)$, obtained after fitting the imaginary part to Eq. (5), for the structural glass and OG. Again, one can see the closeness of behavior below $T_{\mathrm{g}}$ for both phases, as was discussed in the previous section. The solid lines represent the corresponding fits with the Arrhenius law $\tau=\tau_{0} e^{E_{0} / K T}$, where $E_{0}$ is the activation energy of the process. The parameters obtained from the Arrhenius fits for both phases are given in Table I. The graph also shows the $\alpha$ relaxation and its fit to the VTF law.

(ii) In Fig. 9 we plot the temperature dependence of the distribution of energy barriers, $\sigma$, obtained from the peak width $W$. We find that the values for both phases have a roughly linear dependence with temperature, $\sigma=\sigma_{0}-\beta T$, 


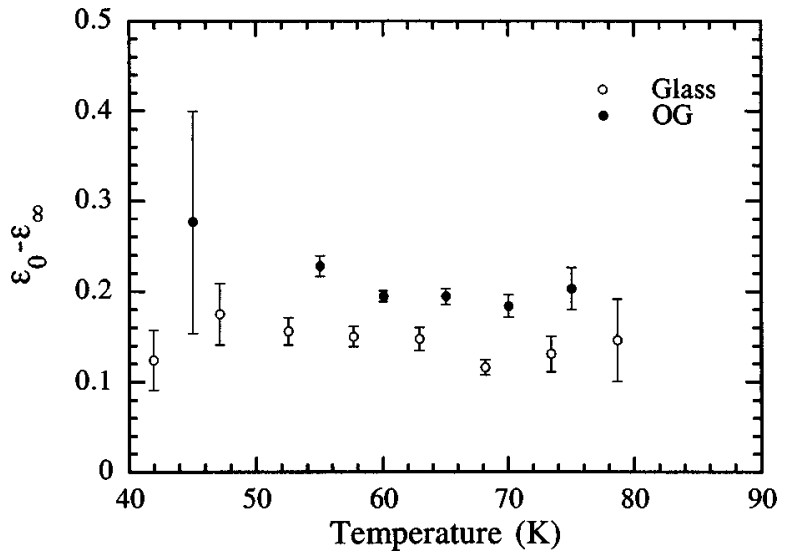

FIG. 10. Temperature dependence of the relaxation strength $\left(\epsilon_{0}-\epsilon_{\infty}\right)$ in the $\beta$ relaxation for the glass and OG phases of ethanol.

where $\sigma_{0}$ is the zero-temperature width of the energy barrier distribution, which we obtain by assuming a linear extrapolation to $T=0 \mathrm{~K}$.

(iii) The temperature dependence of the $\beta$ relaxation strength $\left(\epsilon_{0}-\epsilon_{\infty}\right)$ is shown in Fig. 10. The absolute values for both phases are small if compared with the $\alpha$ relaxation. The $\beta$ relaxation strength in the OG is about 1.3 times larger than in the glass. Such a difference in relaxation amplitudes between the structural and orientational glasses can be understood on the basis of the existence in the latter of a crystalline bcc lattice. To see this one only has to recall that the orientational glass structure is basically a snapshot of that of the RP crystal, and that in the latter molecular rotations are significantly less hindered than in the SCL, as noted above. Upon freezing the large-angle, whole molecule rotations one would expect that segmental and librational motions will take place in the orientational glass phase with less hindrance than in the SCL. This point constitutes a dynamical correlate of what is found in calorimetric measurements of $C_{P}(T),{ }^{8}$ which show that for a range of temperatures below the two transitions $(86 \leqslant T \leqslant 95 \mathrm{~K}) C_{P}^{\mathrm{OG}}>C_{P}^{\text {glass }}$, that is a larger number of particles participate in the motions within the disordered crystal.

\section{DISCUSSION}

At temperatures where the $\alpha$ relaxation dominates the spectrum, the main contribution to the dielectric spectra of both supercooled liquid and plastic-crystalline ethanol should be ascribed to molecular reorientations of some kind. Such an assertion is based upon: (a) the Rietveld analysis of the powder diffraction pattern of the rotator phase crystals which indicate that single molecules reorient quasiisotropically, (b) the comparison of the relaxation times for both SCL and RP referred to above, and (c) results of recent neutron scattering measurements ${ }^{13}$ which show that the rotational melting involves fast motions on a scale of about a picosecond. The result seems to be of relevance for studies of the glass transition, where it is often assumed that the freezing the SCL phase is mostly dominated by the arrest of translational degrees of freedom (i.e., identification of the $\alpha$ relaxation with some flow process). Our results show that reorientational motions play a more important role in freez- ing process than the motions related to mass diffusion which, because of the high viscosities involved, are energetically more costly to execute.

At higher frequencies, the first point to consider is whether the trailing shoulder of the $\alpha$ peak can be sensibly ascribed to an additional relaxation. As stated above, two mutually exclusive rationalizations can be brought to the fore. Our data, although not conclusive, show some evidence signaling a dependence with temperature of the highfrequency wing significantly different from that of the main peak. If this were the case, then the relaxation at such frequencies could possibly be ascribed to some of the sources of relaxation discussed in the past by a number of authors (molecular clusters of varying sizes). ${ }^{19,25,26,29}$ The present findings provide however clear hints showing that such assignments are inadequate. First, the fact that the highfrequency tail appears in the RP seems to invalidate the view $^{25}$ attributing this relaxation to reorientations of free molecules ("monomers" at the end of a hydrogen-bonded "chain'). In fact, as shown previously, ${ }^{8}$ within the RP crystal all molecules must be considered on the same footing, which makes the distinction between "monomers" and associated units of scarce utility. Rather, a combination of mechanisms involved in the $\alpha$ and those for the $\beta$, which are described below, seems physically more sound.

At temperatures well below $T_{\mathrm{g}}$ molecular motions in the glass and OG phases involving whole body rotations are frozen and therefore the dielectric response cannot arise from molecular large-angle reorientations. A way to ascertain the origin of such a relaxation would be to extrapolate the value of its relaxation time up to normal-liquid temperatures where the presence of several relaxation processes has been documented for monohydric alcohols. ${ }^{26}$ In doing so, a value of $1.66 \mathrm{ps}$ is found for room temperature. This value is not too far from the lifetime of a hydrogen bond in water, $\tau_{\mathrm{HB}}$ $=0.54 \mathrm{ps}$, deduced from Rayleigh scattering experiments, ${ }^{32}$ and is very close to that deduced by Barthel et al. ${ }^{26}$ for liquid ethanol at room temperature. In fact values within the interval within 0.22 ps $\leqslant \tau_{3} \leqslant 1.81$ ps have been reported ${ }^{19}$ from studies combining dielectric and infrared spectroscopies where $\tau_{3}$, the shortest relaxation time of the three distinguishable relaxation steps was acribed to the relaxation of the hydroxyl group. On such grounds one can explain why the $\beta$ relaxation is observed at the same frequency for a given temperature in the different phases, but the relaxation strength, $\epsilon_{0}-\epsilon_{\infty}$, is higher in the OG phase.

\section{A. Origin of the observed relaxations}

In what follows a tentative assignment of the relaxations observed within the normal liquid as well as below $T_{\mathrm{g}}$ to underlying molecular motions is proposed. The relaxation dynamics within the supercooled liquid and rotator phase crystals reveals substantially more complicated features than those observed in these two limiting cases.

\section{High-temperature regime}

The presence in liquid monohydric alcohols at temperatures within the normal-liquid range of several dynamical processes which contribute to the quasielastic neutron and light-scattering spectrum (i.e., arising from stochastic mo- 
tions) seems now well established. ${ }^{27,28}$ In fact, several spectral components are needed to account for the observed line shape of the $S_{q . e l}(Q, \omega)$ quasielastic neutron scattering spectrum. The spectrum shows pronounced broadenings of the quasielastic (i.e., centered at zero frequency) peak with linewidths stretching over the experimentally accessible frequency window $(0.25 \mathrm{GHz}-2 \mathrm{THz})$. A comparison with measurements of the high-frequency dielectric response (i.e., measurable by infrared techniques) may be achieved after converting the neutron spectra into generalized susceptibilities, that is $\chi(Q, \omega)=\pi S_{q . e l}(Q, \omega)[1+n(\omega)]$, the term within square brackets being the Bose factor. In addition, computer molecular-dynamics ${ }^{19}$ calculations of the dielectric function for the high-temperature liquid show that the observed spectra may be understood in terms of three distinct relaxation steps ${ }^{26}$ which can be reproduced on semiquantitative grounds using a moderately sized simulation box (about $23 \AA$ on a side).

The presence of several relaxations was rationalized in the past by recourse to some phenomenological models such as that due to Bertolini et al. ${ }^{29}$ which pictures the deviations from ideality (exponential relaxation) of both the rotational and translational diffusion processes as driven by the hydrogen bond dynamics. In the case of dielectric relaxation, the characteristic times are calculated by solution of an equation of motion for the correlation function for the total dipole moment $\Phi_{\mu}^{(i)}$ of a given molecule found in the $i$ th environment. That is, molecules within the sample are assumed to be found in a discrete number of states, which are here interpreted as the number of bonds to a neighboring molecule. Data from computer molecular dynamics ${ }^{17,19,29}$ identify three possible states for these alcohols corresponding to particles with $0-2$ bonds to neighbors (in a time-average sense), respectively. The equation of motion then reads ${ }^{29}$

$$
\frac{d \Phi_{\mu}^{(i)}}{d t}=-D_{\mathrm{R}}\left(\eta_{i}\right) \Phi_{\mu}^{(i)}(t)+\sum_{j=0,}^{2}\left(K_{\eta}\right)_{i j} \Phi_{\mu}^{(j)}(t), \quad i=0,2,
$$

where $D_{\mathrm{R}}\left(\eta_{i}\right)$ stands for the rotational diffusion coefficient for molecules in the $i$ th state, and $\left(K_{\eta}\right)_{i j}$ is a matrix of kinetic coefficients connecting the states $i-j$ which is given in terms of the probabilities for each $i$ th state. The relevant parameters to specify such transition rates are the "hydrogen-bond" lifetime $\tau_{\mathrm{HB}}$, the time $\tau_{f}$ which one bond may last as broken and finally the probabilities $p_{0,2}$ of molecules in each state. All such parameters are in principle obtainable from molecular simulations and, as we will see below, only a rough estimate of them is required to interpret the general trends of the dielectric relaxation spectra.

Solution of Eq. (7) is easily achieved in terms of the eigenvalue equation $^{29}$

$$
\operatorname{det}||\left[D_{\mathrm{R}}\left(\eta_{i}\right)-\lambda+K_{\eta}\right] I||=0
$$

from where the relaxation times are determined from the calculated eigenvalues.

An assessment of the capability of Eq. (8) to reproduce the most salient features of the dielectric spectrum was carried out using present results supplemented with the high- frequency data for room-temperature ethanol reported by Barthel et al. ${ }^{26}$ The required values for $p_{0,2}$ and $\tau_{\mathrm{HB}}$ are taken from molecular dynamics results, ${ }^{19,29}$ and rough estimates for the rotational coefficients for each $i$ th state $D_{\mathrm{R}}\left(\eta_{i}\right)$ were derived on hydrodynamic grounds, that is using the StokesEinstein-Debye formula for the corresponding molecular volumes, temperature, and viscosity. Using the constraint $\bar{D}_{\mathrm{R}}=\Sigma_{i=0,2} f_{i} D_{\mathrm{R}}\left(\eta_{i}\right)$, where $\bar{D}_{\mathrm{R}}$ was identified with the experimental value for the rotational coefficient measured by NMR (Ref. 33) enables one to put the hydrodynamic estimates into an absolute scale. There, $f_{i}$ stands for the fraction of molecules in a given state and the only free parameter left is $\tau_{f}$. Solution of Eq. (8) with such parameters leads to an estimate of the three relaxation times as $160,3.5$, and $1.8 \mathrm{ps,}$ which compares with $163,8.97$, and $1.91 \mathrm{ps}$, as reported in Ref. 26. That is, it correctly predicts the relaxation time of the main $\alpha$ dielectric band in the high-temperature liquid, showing that it roughly coincides with the time spent by a molecule in the structured part of the liquid, and accounts for the presence of additional relaxation processes.

Such a picture, although physically appealing, loses its validity within the deeply supercooled liquid. In fact, at temperatures well below $\approx 140 \mathrm{~K}$ the predicted behavior of $\tau(T)$ for the $\alpha$ relaxation lies substantially below experiment. The interesting point is that this temperature, which also marks the limit of stability of the supercooled liquid, signals a strong departure from Arrhenius behavior of a number of momentum and charge transport properties. ${ }^{34}$ The model cannot follow the strong temperature dependence of $\tau(T)$ but still predicts the presence of additional relaxations which show an Arrhenius behavior of their frequencies, and show characteristic residence times $\tau_{\mathrm{HB}}$ within 100-350 ps. The failure of Eq. (8) to account for the dynamics of the SCL is understood as a breakdown of the validity of the approximations upon which such treatment was built. In a nutshell, it simply illustrates the breakdown of the Browniandynamics regime, a departure which is also witnessed by several transport properties. ${ }^{34}$

\section{2. $\beta$ relaxation}

The most striking results coming from the measurements below the two glass transitions concern the closeness of the activation energies $E_{0}$ and widths of the barrier distributions $\sigma$ for both the structural and orientational glasses. The latter quantities can be extrapolated to $T=0 \mathrm{~K}, \sigma(T)=\sigma_{0}-\beta T$ yielding values of 14.5 and $11.1 \mathrm{THz}$, respectively. Such figures and especially the ratios $\sigma_{0} / \mathrm{E}_{0}=0.74$ and 0.45 are comparable to others measured in the glassy crystal, such as $(\mathrm{KBr})_{1-x}(\mathrm{KCN})_{x}$ with "defect" concentrations within its "glassy" range. ${ }^{35}$ Motivated by this analogy, we have followed the steps delineated by Grannan et al. ${ }^{36}$ with the aim of identifying the microscopic entities giving rise to frequency dependent dielectric signals.

Well below the glass transitions, rigid body molecular reorientations involving large amplitude angular excursions which would give rise to dielectric response arising from motions of the molecular dipoles seem highly unlikely. Instead, the motions involving some molecular segment carrying a large local dipole moment may indeed take place as a consequence of a thermally activated mechanism. From mi- 
croscopic modeling of the dynamics of the solids whether in orientationally ordered, orientationally disordered, or glassy phases ${ }^{10}$ the two most likely candidates to give rise to lowfrequency motions not involving large rearrangements of neighboring particles are torsional oscillations about the $\mathrm{C}-\mathrm{C}$ and $\mathrm{C}-\mathrm{O}$ molecular bonds. The characteristic harmonic frequencies for such librations were estimated from $a b$ initio Hartee-Fock calculations as 7.3 and $9.1 \mathrm{THz}$, respectively, and found to correspond to definite features in the experimental densities of states. ${ }^{10}$ Although those frequencies are far too high to contribute to relaxation within the $10^{-8}-10^{-3}$ s scale, the point which merits attention concerns the strong hybridization between these internal and "lattice" motions which will provide a coupling between these and low-frequency vibrations such as long-wavelength hydrodynamic phonons. Such hybridization occurs as a consequence of the lack of time-scale separation between lowenergy molecular and phonon modes (in fact, the experimental density of states up to $20 \mathrm{THz}$ lacks any clear gap ${ }^{10}$ ). Explicit evaluation of such effects in a glassy material ${ }^{37}$ shows how hybridization alters dramatically the density of vibrational states of a solid composed by rigid molecules. Indeed, the resulting frequency distribution is remarkably stretched towards low and high frequencies. In other words, such a mode mixing results in a large increase of the vibrational density of states at low frequencies which arises from the admixture of acoustic phonons with the molecular internal motions. The latter thus become strongly coupled to the strain fields and consequently, any excitation of such fields taking place at relatively low frequency is expected to involve a substantial molecular deformational (i.e., involving low-energy molecular internal coordinate) component. Such a mechanism is thus able to explain why the relaxations sampled at macroscopic scales can follow the motions of these high-frequency modes.

Between the two plausible candidates referred to above, only motions along the $\mathrm{C}-\mathrm{O}$ bond are expected to be active in a dielectric relaxation experiment because of the relatively strong $\mathrm{OH} \rightarrow \mathrm{C}$ local dipole. If this were the case then the harmonic frequency given above should not be far from $\omega_{\text {harm }}=\sqrt{\left(2 E_{0} / I\right)}$, where $E_{0}$ stands for the barrier height and $I$ is the effective moment of inertia of the reorienting entity. Using data from Table I for $E_{0}$ and the value for the moment of inertia of the free molecule, $I=2.84$ $\times 10^{-45} \mathrm{Kg} \mathrm{m}^{2}$ one gets $\omega_{\text {harm }}$ frequencies of 3.06 and 3.18 $\mathrm{THz}$ for the glass and OG crystal, respectively. The role of a quantity such as $\omega_{\text {harm }}$ is to provide a rational basis to understand the origin of the large values for the $f_{0}$ " attempt frequencies" given in Table I. Using the same arguments as those given in Ref. 35, one finds that the "attempt frequencies" $f_{0}$ which are about $2.4 \mathrm{THz}$ for the glass and $10 \mathrm{THz}$ for the glassy crystal, can be qualitatively interpreted in terms of the characteristic frequency of some microscopic process, such as the libration of some molecular dipole. On such a basis, we have evaluated two of the most relevant functions which will give rise to finite frequency peaks in the dielectric function. They are the librational frequency distributions (calculated from the autocorrelation of angular velocities) of a vector parallel to the total molecular dipole moment $P_{\mu}(\omega)$, and of a vector parallel to the $\mathrm{O}-\mathrm{H}$ bond $P_{\mathrm{OH}}(\omega)$. The results are shown in Fig. 11.

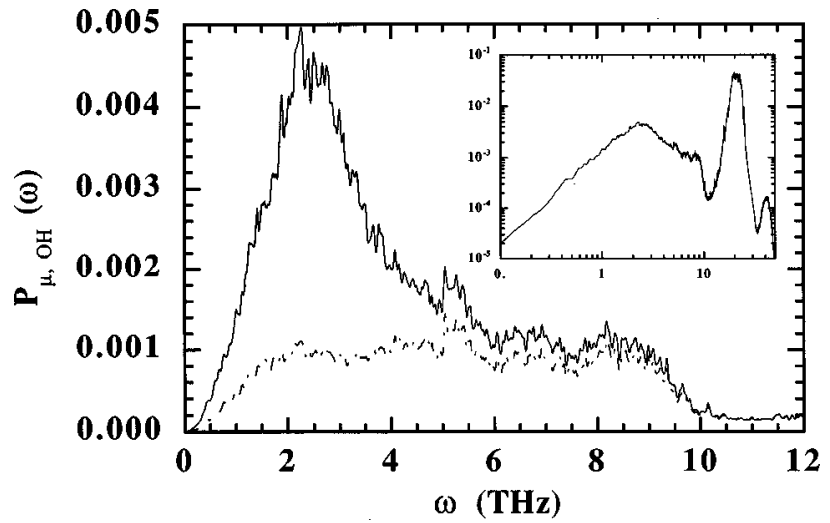

FIG. 11. The low-frequency part of the $P_{\mu}(\omega)$ and $P_{\mathrm{OH}}(\omega)$ distributions of librational frequencies for a vector directed along the molecular dipole (solid line) and along the $\mathrm{O}-\mathrm{H}$ bond (dashes), respectively. The glass $(80 \mathrm{~K})$ and supercooled liquid $(180 \mathrm{~K})$ show very close frequency distributions. The inset shows the distribution for the whole range of frequencies.

A glance at the graphs shown in Fig. 11 unravels the strong mixing of modes referred to above. In other words, although the strongest peak appearing in $P_{\mu}(\omega)$ and $P_{\mathrm{OH}}(\omega)$ are located at fairly high frequencies (about $20 \mathrm{THz}$ ), a broad band is seen at low frequencies as a result of the strongly collective nature of most motions taking place in these phases. That is, most motions including those below $1 \mathrm{THz}$ which are dominated by long-wavelength phonons are strongly coupled to movements which will generate a response of the polarization field. As expected, both $P_{\mu}(\omega)$ and $P_{\mathrm{OH}}(\omega)$ show well defined maxima within frequencies covered by the broad maxima of the vibrational frequency distributions. ${ }^{12}$ Such a situation is reminiscent of that of mixed $\mathrm{KBr}: \mathrm{KCN}$ halide crystals $^{36}$ where harmonic excitations in addition to acoustic phonons are found. These show a density of states $g_{l i b}(\omega)$ peaked at about $1 \mathrm{THz}$ and correspond to librations of the $\mathrm{CN}$ group, whose presence serves to explain the peak in $C_{P}(T) / T^{3}$, the low-temperature specific heat, and the "plateau" in the thermal conductivity. Such "additional harmonic excitations" are also well known from studies on canonical glasses such as vitreous $\mathrm{SiO}_{2}{ }^{38}$ and indeed, show a peaked density of states at about $2 \mathrm{THz}$.

The shape of both $P_{\mu}(\omega)$ and $P_{\mathrm{OH}}(\omega)$ displayed in Fig. 11 shows the same extrema albeit with rather different weights. This should not come as a surprise in view of the strong contribution of the $\mathrm{O}-\mathrm{H}$ fragment to the molecular dipole moment. The $P_{\mu}(\omega)$ distribution seems however more meaningful if the same arguments given in Ref. 36 are brought to the fore. That is, if one identifies $P(\omega)$ as the function giving rise to the maxima in $C_{P}(T) / T^{3}$ at about 5-7 $\mathrm{K},{ }^{10}$ then it should show a low frequency part not too different from that shown by the total density of states $Z(\omega){ }^{10,12}$ On such a basis, the $P_{\mu}(\omega)$ distribution seems to be the sought quantity.

\section{Dynamics of the supercooled liquid}

The fact that the dynamics of the bcc crystal lies so close to that of the SCL serves to scrutinize the various explanations offered in the literature ${ }^{3}$ concerning the origin of the main relaxation. These go from those assuming individual 


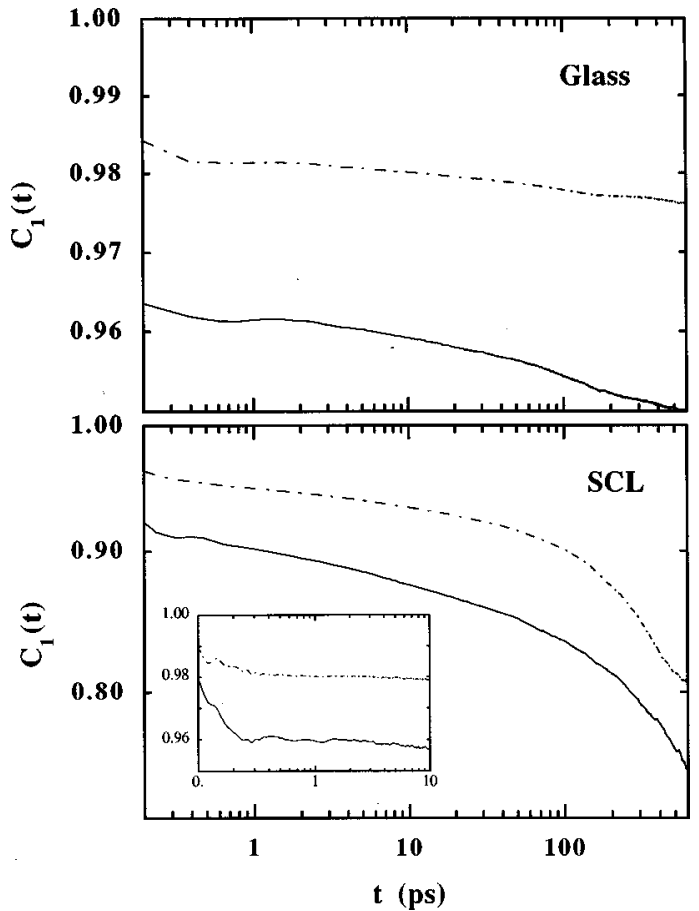

FIG. 12. The $C_{1}(t)$ reorientational correlation functions derived from the computer molecular-dynamics calculations. Solid traces show the reorientation of a vector $\boldsymbol{\mu}$ directed along the molecular dipole moment and dot-dashes depict the function for a vector located along the $\mathrm{O}-\mathrm{H}$ bond. The inset shows an enlargement of the decay at short times.

molecular dipole reorientations to those involving molecular clusters. $^{39}$ The former possibility is ruled out by consideration of data from NMR and neutron quasielastic scattering measurements ${ }^{13,14,40}$ which show that within the rotator crystal whole molecule quasi-isotropic ${ }^{8}$ reorientations within the picosecond scale take place (about ten orders of magnitude faster than those sampled by dielectric measurements). On the other hand the latter seems wholly incompatible with the very existence of the crystalline bcc lattice.

To understand the origin of the relaxation under high viscosity conditions some help can be sought from the calculated computer molecular dynamics trajectories. Specifically, we aim to derive some relevant conclusion from comparison between the reorientational correlation functions

$$
C_{l}(t)=\left\langle P_{l}[\cos \theta(t)]\right\rangle
$$

at both sides of the glass transition. Here, $P_{l}$ stands for the $l$ th Legendre polynomial and $\theta(t)$ is the angle through which a molecule fixed vector (either along $\boldsymbol{\mu}$ or the $\mathrm{O}-\mathrm{H}$ bond) rotates in time $t$. Figure 12 provides such a comparison. There, the functions $C_{1}(t)$ which are relevant for dielectric relaxation are shown for the two vectors referred to above for $T=80 \mathrm{~K}$ (glass) and $T=180 \mathrm{~K}$ (still in the supercooled liquid range for the computer glass). All the curves shown there exhibit an initial fast decay with superimposed oscillations which last about 0.5 ps followed by a flat or a slowly decaying regime stretching to several tens of picoseconds. As a main distinctive feature of the SCL state a second strong decay is seen after about 100 ps or so. Again, the shape of
$C_{1}(t)$ for both $\boldsymbol{\mu}$ and $\mathrm{O}-\mathrm{H}$ vectors is not too different, exception made of the absolute values as well as of a stronger initial decay of the latter.

The results shown in Fig. 12 thus illustrate the presence of different time scales in the orientational dynamics of this material. The strong decay at long times appearing in the SCL can be tentatively identified with the tail of the main $\alpha$ relaxation (the simulations are too short to sample the required time scales) whereas the region at intermediate times (1-100 ps), which is common to SCL and glass should be related to the higher frequency relaxation. Its slow decay will translate into a broad spectrum in the frequency domain which will be very weak if compared with that originated by the decay at long times. In fact, the Fourier transforms of the correlation functions show three separate regions corresponding to a low-frequency broad band which dominates the spectrum, a second region stretching from about 1-10 $\mathrm{THz}$, and a well defined microscopic peak at a higher frequency.

\section{CONCLUSIONS}

The dielectric measurements of the $\alpha$ relaxation have demonstrated that although the glass transition is regarded as a structural transition, the glasslike transition in RP (with translational order) exhibits all the characteristics observed in the canonical transition SCL $\rightarrow$ glass: a decrease of the width parameter as the glass transition is approached and a relaxation described by the Vogel-Tamman-Fulcher form. The relaxation times above $96 \mathrm{~K}$ display the effect of the additional translational freedom available to the liquid resulting in faster molecular reorientation in the SCL relative to the RP crystal for a given temperature. We can therefore conclude that orientational relaxations play an important role in the glass transition which has been considered up to recent times as a purely translational transition.

In addition, our results serve to illustrate how molecular motions of different kinds (i.e., pure reorientations and mass diffusion) exhibit disparate temperature dependences when the macroscopic viscosity reaches the large values characteristic of the supercooled liquid. This can be viewed as a transition from a high-temperature regime where collective effects are reduced to distances comparable to few particle diameters, to another where such motions involve meso- or macroscopically large volumes. One then expects that the Brownian dynamics approximation (i.e., same temperature dependence for all the diffusions coefficients) will only hold within the normal-liquid phase. To put it somewhat differently, the large number of degrees of freedom which are thermally activated in the latter phase are seen as a "thermal bath" by the reorienting dipoles. In contrast, within the SCL and lower temperature phases collective effects are felt with increasing strength as the temperature is lowered leading to a complete absence of separation of the time scales characteristic of "fast" (i.e., the thermal bath) and "slow" motions.

The results also lend additional support to the idea put forward from recent studies, ${ }^{10-12}$ which attributes a purely dynamic character to the $\mathrm{RP} \rightarrow \mathrm{OG}$ transition. This makes the orientationally disordered crystals ideal candidates for the study of the glass transition, and also enables one to draw a parallelism with the well studied cases of orientational 
glasses. ${ }^{41}$ In both cases, the presence of long order positional periodicity enables one to understand the freezing phenomenon on the basis of some minimal model, as that of hard needles on a fcc lattice, explored by Renner et al. ${ }^{42}$ It contains the basic ingredients of the "glass transition." Indeed, it exhibits a kinetic glass transition when $l=L / a$, the needle length to lattice constant ratio, exceeds about 2.7 , and is simple enough to a allow numerical and analytical study. For a bcc lattice, such as that of the RP and OG crystal phases, a "computer glass transition" is observed for a ratio of $3.3-3.4{ }^{13}$

The origin of the $\beta$ relaxation is ascribed to thermally activated local molecular librations. Our analysis shows that such motions are the result of a strong coupling between molecular internal and collective modes, and some points of contact are established with work performed on $\mathrm{KBr}: \mathrm{KCN}$ (Ref. 36) mixed crystals where the flipping motion of the $\mathrm{CN}$ dipoles is postulated as the origin of this relaxational peak. The intensity of the $\beta$ relaxation is higher in the OG than in the structural glass because in the fully disordered phase the molecular orientations are strongly correlated with the positions of the molecular centers of mass. This result agrees with the excess in specific heat $C_{P}$ observed in previous work, in which the observed $C_{P}$ below the glass transition in the OG is higher than in the glass. ${ }^{8}$

Finally, one of the most interesting aspects in the comparison of the dynamical behavior of the glassy and disordered crystal states of this material would be to compare the temperature and frequency dependence of their elastic constants, which are expected to show marked anomalies with respect to those shown by the ordered crystal.

\section{ACKNOWLEDGMENTS}

This work was performed in part under DGICYT (Spain) Grant Nos. PB95-0075-C03-01 and PB94-0468. We thank R. Fernández-Perea for helpful discussions.
${ }^{1}$ H. Vogel, Z. Phys. 22, 645 (1961); G.S. Fulcher, J. Am. Chem. Soc. 8, 339 (1925); G. Tamman and W. Hesse, Z. Anorg. Allg. Chem. 156, 245 (1926).

${ }^{2}$ L. Wu, Phys. Rev. B 43, 9906 (1991).

${ }^{3}$ A. Kudlik, C. Tschirwitz, S. Benkhof, T. Blochowicz, and E. Rössler, Europhys. Lett. 40, 649 (1997).

${ }^{4}$ Dynamics of Disordered Materials II, edited by A.J. Dianoux, W. Petry, and D. Richter (North Holland, Amsterdam, 1993).

${ }^{5}$ R. Schilling and T. Scheidsteger, Phys. Rev. E 56, 2932 (1997); S. Kämmaner, W. Kob, and R. Schilling (unpublished).

${ }^{6}$ M. Evans, G.J. Evans, W.T. Coffey, and P. Grigolini, Molecular Dynamics and Theory of Broadband Spectroscopy (Wiley, New York, 1982), Chap. 2.

${ }^{7}$ O. Haida, H. Suga, and S. Seki, J. Chem. Thermodyn. 9, 1133 (1977); M. Hugo, K. Schlüter, and A. Würflinger, Z. Phys. Chem. (Munich) 175, 235 (1992).

${ }^{8}$ F.J. Bermejo, A. Criado, R. Fayos, R. Fernández-Perea, H.E. Fischer, E. Suard, A. Guelylah, and J. Zúñiga, Phys. Rev. B 56, 11536 (1997); F.J. Bermejo, H.E. Fischer, M.A. Ramos, A. de Andrés, J. Dawidowski and R. Fayos, in Complex Behaviour of Glassy Systems, edited by M. Rubí and C. Pérez-Vicente, Springer Lecture Notes in Physics, Vol. 492 (Springer, Berlin, 1997), p. 44.

${ }^{9}$ R. Fayos, F.J. Bermejo, J. Dawidowski, H.E. Fischer, and M.A. González, Phys. Rev. Lett. 77, 3823 (1996).

${ }^{10}$ C. Talón, M.A. Ramos, S. Vieira, G.J. Cuello, F.J. Bermejo, A. Criado, M.L. Senent, S.M. Bennington, H.E. Fischer, and H. Schober, Phys. Rev. B 58, 745 (1998).

${ }^{11}$ H.E. Fischer, F.J. Bermejo, G.J. Cuello, M.T. Fernández-Díaz, J. Dawidowski, M.A. González, H. Schober, and M. JiménezRuiz, Phys. Rev. Lett. 82, 1193 (1999).

${ }^{12}$ F.J. Bermejo, G.J. Cuello, J. Dawidowski, H.E. Fischer, H. Schober, M.A. González, and S.M. Bennington, Physica B 241243, 883 (1998).

${ }^{13}$ M.A. Ramos, S. Vieira, F.J. Bermejo, J. Dawidowski, H.E. Fischer, H. Schober, M.A. González, C.K. Loong, and D.L. Price, Phys. Rev. Lett. 78, 82 (1997).

${ }^{14}$ M. Miller, M.A. Jiménez-Ruiz, F.J. Bermejo, and N.O. Birge,
Phys. Rev. B 57, R13 977 (1998).

${ }^{15}$ D.L. Leslie-Pelecky and N.O. Birge, Phys. Rev. B 50, 13250 (1994); Phys. Rev. Lett. 72, 1232 (1994).

${ }^{16}$ M.A. González, E. Enciso, F.J. Bermejo, and M. Bée, J. Chem. Phys. (to be publsihed).

${ }^{17}$ W.L. Jorgensen, J. Phys. Chem. 90, 1276 (1996).

${ }^{18}$ L. Saiz, J.A. Padró, and E. Guàrdia, J. Phys. Chem. B 101, 78 (1997); also, L. Saiz, Ph.D. dissertation, University of Barcelona, 1998.

${ }^{19}$ D.W. Davidson and R.H. Cole, J. Chem. Phys. 18, 1417 (1951).

${ }^{20}$ P.K. Dixon, L. Wu, S.R. Nagel, B.D. Williams, and J.P. Carini, Phys. Rev. Lett. 65, 1108 (1990).

${ }^{21}$ P. Lunkenheimer, A. Pimenov, M. Dressel, Y.G. Goncharov, R. Böhmer, and A. Loidl, Phys. Rev. Lett. 77, 318 (1996).

${ }^{22}$ R.H. Cole, in Molecular Liquids-Dynamics and Interactions, edited by A.J. Barnes, W.J. Orville-Thomas, J. Yarwood, Vol. 135 of NATO Advanced Study Institute, Series C: Mathematical and Physical Sciences (Reidel, Dordrecht, 1983), p. 91.

${ }^{23}$ M.A. Miller, Ph.D. thesis, Michigan State University, 1998.

${ }^{24}$ S.K. Garg and C.P. Smyth, J. Phys. Chem. 69, 1294 (1965).

${ }^{25}$ J. Barthel, K. Bachhuber, R. Buchner, and H. Hetzenauer, Chem. Phys. Lett. 165, 369 (1990). For recent results see J.T. Kindt and C.A. Schmuttenmaer, J. Phys. Chem. 100, 10373 (1996).

${ }^{26}$ F.J. Bermejo, F. Batallán, W.S. Howells, C.J. Carlile, E. Enciso, M. Garc-Hernández, M. Alvarez, and J. Alonso, J. Phys.: Condens. Matter 2, 5005 (1990).

${ }^{27}$ M.P. Janelli, S. Magazú, P. Migliardo, F. Alliotta, and E. Tettamanti, J. Phys.: Condens. Matter 8, 8157 (1996).

${ }^{28}$ D. Bertolini, M. Cassettari, M. Ferrario, P. Grigolini, and G. Salvetti, Adv. Chem. Phys. 62, 277 (1985).

${ }^{29}$ L. Wu, R.M. Ernst, Y.H. Jeong, S.R. Nagel, and S. Susman, Phys. Rev. B 37, 10444 (1988).

${ }^{30}$ N.O. Birge, Y.H. Jeong, S.R. Nagel, S. Bhattacharya, and S. Susman, Phys. Rev. B 30, 2306 (1984).

${ }^{31}$ M. Jiménez-Ruiz, G.J. Cuello, F.J. Bermejo, F.R. Trouw, A. Criado, and H.L. Löwen (unpublished).

${ }^{32}$ O. Conde and J. Teixeira, J. Phys. (Paris) 44, 525 (1982).

${ }^{33}$ R. Ludwig and M.D. Zeidler, Mol. Phys. 82, 313 (1994). 
${ }^{34}$ F. Stickel, E.W. Fischer, and R. Richert, J. Chem. Phys. 104, 2043 (1996).

${ }^{35}$ R.M. Ernst, L. Wu, S.R. Nagel, and S. Susman, Phys. Rev. B 38, 6246 (1988).

${ }^{36}$ E.R. Grannan, M. Randeria, and J.P. Sethna, Phys. Rev. B 41, 7784 (1990); 41, 7799 (1990).

${ }^{37}$ A. Criado, F.J. Bermejo, A. de Andrés, and J.L. Martínez, Mol. Phys. 82, 787 (1994).

${ }^{38}$ U. Buchenau, M. Prager, N. Nucker, A.J. Dianoux, N. Ahmad, and W.A. Phillips, Phys. Rev. B 34, 5665 (1986).

${ }^{39}$ C.A. Angell, in Hydrogen-Bonded Liquids (Kluwer Academic, Dordrecht, 1991), p. 59.

${ }^{40}$ T. Eguchi, G. Soda, and H. Chihara, Mol. Phys. 40, 681 (1980).

${ }^{41}$ A. Loidl, in Disorder Effects on Relaxation Processes, edited by R. Richert and A. Blumen (Springer, New York, 1994).

${ }^{42}$ C. Renner, H. Löwen, and J.L. Barrat, Phys. Rev. E 52, 5091 (1995). 